\title{
RIATTIVAZIONE DI EPATITE VIRALE B IN \\ PAZIENTE CON ANTICORPI ANTI S A TITOLO ELEVATO, DOPO TERAPIA CON RITUXIMAB
}

Pedroni M., Milanesi B.

Laboratorio di Patologia Clinica

Azienda Ospedaliera Desenzano del Garda,

Presidio di Manerbio, BS

Direttore: Prof. Milanesi B.

Introduzione. Riscontro di Epatite virale B acuta, in paziente con valori precedenti di anticorpi anti $\mathrm{S}(\mathrm{HBsAb})$ superiori a $1.000 \mathrm{IU} / \mathrm{L}$, dopo terapia con anticorpi chimerici antiCD20 (Rituximab) per Linfoma non-Hodgkin (NHL).

Descrizione del caso. Paziente di 58 anni si presenta nel marzo 2005, presso il Centro Prelievi Ambulatoriali del nostro Laboratorio per eseguire esami di controllo per ipertransaminasemia, AST 119 UI/L, ALT 319 UI/L. Il quadro sierologico per il virus dell'Epatite B, presenta i seguenti valori: $\mathrm{HBsAg}+, \mathrm{HBsAb}-, \mathrm{HBcAb}+, \mathrm{HBeAg}+, \mathrm{HBeAb}-$, compatibili con una infezione recente da Virus B. Il controllo di delta-check in linea, in uso presso il nostro Laboratorio, evidenziava un precedente riscontro, novembre 2003, di 
HBsAg- e HBsAb > 1.000 IU/L. Dopo ricontrollo su nuovo prelievo, veniva approfondita la storia clinica del paziente. L'emocromo, eseguito nel novembre 2003, sempre presso il nostro ambulatorio, evidenziava alcuni linfociti con atipie morfologiche, atipia che richiedeva un approfondimento diagnostico; l'approfondimento, eseguito presso altro Ospedale, portava ad una diagnosi di NHL. Gli accertamenti sierologici eseguiti al ricovero nel Dicembre 2003, confermavano la negatività dell'HBsAg. Accertamenti successivi, settembre 2004, sempre presso altro Ospedale confermavano 1' HBsAg-e HBsAb di $95 \mathrm{UI} / \mathrm{L}$. Il paziente è stato trattato con Rituximab per il NHL.

Conclusioni. La segnalazione del caso, vuole richiamare l'attenzione sui seguenti punti:

- Riattivazione di Epatite virale B, in presenza di Anticorpi anti $\mathrm{S}$ a titolo elevato ed HBsAg-, dopo terapia con Rituximab.

- Importanza di un controllo di delta-check in linea per il monitoraggio dell'evoluzione della sierologia virale, da parte del Laboratorio.

- Nessun riscontro di segnalazioni su MedLine di casi Italiani di riattivazione di Epatite B.

- Numerose segnalazioni internazionali (14 su MedLine ad aprile 2005), con prima segnalazione nel 2001.

- Importanza della profilassi per l'Epatite B in caso di terapia con Rituximab.

\section{BIBLIOGRAFIA}

1. Servite I., Hober D., Morel P., Acute Hepatitis B in a patient with antibodies ta hepatitis B surface antigen who was receiving Rituximab. N Engl J Med 2001; 344:68-69. 